MATEC Web of Conferences 43, 01001 (2016)

DOI: $10.1051 /$ matecconf/20164301001

(C) Owned by the authors, published by EDP Sciences, 2016

\title{
Simulation Techniques for Porous Media Confined in Silo Geometry
}

\author{
Dr. Abdul Qadir ${ }^{1, a}$, Kashif Hussain Memon ${ }^{1}$ \\ ${ }^{1}$ Department of Electrical Engineering, Sukkur Institute of Business Administration, Sukkur, 65200, Pakistan.
}

\begin{abstract}
Granular materials have vast applications both in industry and in daily life. They display quite interesting and exceptional properties different from the other known forms of matter. To investigate the complex properties of particulate materials, experimental, analytical and simulation techniques have been employed. In this paper the results of large-scale discrete element molecular dynamics (DEM) simulations in three dimensions are reported. It deals with the investigation of stress deflection due to various grain sizes. Moreover, the influence of coefficient of friction between granules and cylindrical walls on the mass measured at bottom of container i.e. apparent mass is thoroughly investigated. It is revealed that apparent mass varies linearly with the grain size. In addition to that, it is also found that the apparent mass variation is strongly dependent on bead diameter rather than the silo. The results reveal that conversion of vertical stresses into horizontal in silo is mainly due to the friction between the grain and system boundary.
\end{abstract}

\section{Introduction}

Granular packing has long been of interest to engineering and scientific community [1]. Since granular materials are ubiquitous, therefore any increased understanding can lead to numerous benefits $[2,3]$. However, the behaviour of granular media in static state has not been fully understood. This results in problems at design stage of silos that leads to expected silo failure. Nowadays silo geometry has emerged as an important tool to study the behaviour of granular media. By studying the form of force measured at the bottom much can be inferred about the stress distribution and propagation in granular systems.

The pioneering work on silo geometry was carried out by Janssen, it was demonstrated that mass measured at the base of a granular pile, known as apparent mass, do not vary linearly with the height of column rather it saturates exponentially [4]. This is in quite contrast with the case of hydrostatics. Vanel etal devised an experimental set up with an aim to measure the pressure profile of a slowly descending granular pile. Furthermore, their investigations exhibited some differences between their experiments and the model. Therefore, a new model was proposed known as

Oriented stresses linearity model [13]. However, subsequent studies have shown that the Janssen model gives

more accurate results compared with other silo models [12]. Extensive studies have been carried out regarding the Janssen model however, few of them focuses the dependence of apparent mass measurement on the friction between silo wall and confined materials, due to inherent experimental difficulties.

In order to circumvent this problem computer simulation has appeared as an important tool to investigate the stresses in confined granular media. Numerous methods have been evolved to investigate granular media in the two dimensional systems [4, 5]. However, the prediction of these models and the approach to take the accurate simulation differs $[6,7]$. In three dimension (3D) simulation usually the discrete element methods is employed. Unfortunately the methods provide a little insight about the internal structure and forces in granular packing $[8,9]$. However, in the case of $3 \mathrm{D}$ simulation, using periodic boundary conditions in two directions perpendicular to the gravity have mainly focused on the internal structure of the confined media $[10,11]$.

In this paper using large-scale discrete element molecular dynamics (DEM) simulations in 3D the dependence apparent mass and coefficient of friction between silo walls have been investigated. The apparent mass is measured systemically by altering the friction values. It is found that apparent mass variation is also strongly dependent on coefficient of static friction between beads and silo wall, rather than the arching phenomenon only as usually believed [19].

\section{Simulation method}

We have used Discrete Element Method (DEM), this method was originally developed by Cundal and Strack with an aim to investigate the mechanical behavior of

\footnotetext{
${ }^{\mathrm{a}}$ Corresponding author: aqadir@iba-suk.edu.pk
} 
spheres and disks [6-8]. In this paper the simulation rests on the Discrete Element Method code "LIGGGHTS" initially employed by Kloss et al. [15, 16]. The code is enhanced edition of the granular package of classical MD simulator LAMMPS [17] and is executed in a new Hertz-Mindlin granular contact model. The model assumes that the interactions among beads and confining boundary are like spring-dashpot in the perpendicular and parallel directions to their lines of centers.

For two adjacent beads "i" and "j" represented by position vectors ri and $\mathrm{rj}$ experience a relative normal compression, given by

$$
\delta=\left|r_{i j}-d\right|, \text { where } r_{i j}=r_{i}-r_{j},
$$

the force so produced is given by

$\mathrm{Fnm}=\mathrm{Fn}+\mathrm{Ft}$

$$
F_{n m}=F_{n}+F_{t}
$$

the normal force is represented by $F$ :

$$
F_{n}=f(\delta / d)\left(k_{n} \delta n_{i j}-\frac{m}{2} \gamma_{n} v_{n}\right) \ldots \ldots .
$$

and tangential force by $\mathrm{Ft}$ :

$$
F_{t}=f(\delta / d)\left(-k_{t} \Delta s_{t}-\frac{m}{2} \gamma_{t} v_{t}\right)
$$

Whereas $v_{n}$ and $v_{n}$ represent the relative surface velocity's normal and tangential components, " $\mathrm{m}$ " is the mass of particles and $\Delta s_{t}$ is elastic tangential displacement among grains. While $k_{n, t}$ and $\gamma_{n, t}$ are elastic and viscoelastic constants, respectively. For Hertzian contacts, $f(x)=\sqrt{x}$. The friction between beads denoted by $\mu \mathrm{b}$ has been kept constant i.e. 0.5 .

This paper presents two modes of simulations. In the first series of simulation, keeping the grain diameter constant $\mathrm{i}$-e $\mathrm{d}=0.20$ units. While friction between the particle and system boundary $\mu$ is varied is changed as $0.20,0.40,0.60,0.80$ and 1.00 . The convenient time unit $\tau=\sqrt{d / g}$ is adopted. Here, time-step of $\delta t=$ $10^{-4 \tau}, k_{n}=4 \times 10^{5} \mathrm{mg} / \mathrm{d}$ and $k_{t}=2 / 7 k_{n}$ and damping coefficients $\gamma \mathrm{n}=\gamma \mathrm{t}=1000 \sqrt{d / g}$ are used. In the next series of simulation, keeping the friction coefficient $\mu$ constant the grains diameter is varied from $d=2.0$ to $4.0,5.0,6.0$, in every simulation run the silo size is kept constant. All simulation has been carried out with fixed set of parameters and measurement of quantities is in dimensionless units based on "m", "g", and "d".

In these simulations, granular packing has been generated by the method of pouring $\mathrm{N}$ mono-disperse beads from a fixed height of $z=280$ units. The height of granular column is set equivalent to 6 times the diameter of silo. The packing is allowed to rest under gravity. In the beginning volume fraction was found to be 0.13 and finally reaches $\sim 0.615$. The model and techniques can be found in contemporary research [1517]. The simulations were run until the kinetic energy per bead became the least. It is achieved by descending the base of silo. Such packing, known as quiescent, has been adopted for further investigation. Pilings are examined before and after cessation of base movement.

Figure 1 shows the structure of a packing with base velocity $(0.001 \mathrm{~d} / \tau)$ in the downward direction $(-\mathrm{z})$. Then notable particle rearrangement takes place during the descending of pile. Many beads initially in touch with the confining boundary moved upward. Moving beads are coloured according their velocities in order to give a better visualization of beads movement as time elapses. During motion, the packing height does not change.

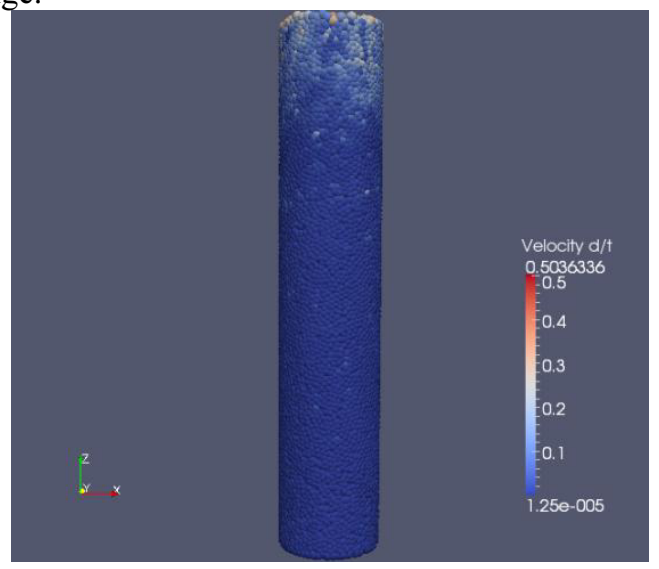

Figure 1. Snapshot of simulation of granular packing

\section{Results and discussion}

Fig. 2 shows the variation in apparent mass measurement with the filling mass. It is revealed that for lower filling mass values the granular material exhibits behavior similar to the hydrostatic case. However, for higher filling mass values the apparent mass becomes constant and a saturated state is attained. It may be noted that stress curves augment and then asymptote. Moreover, the stress curves diverge with bead size.

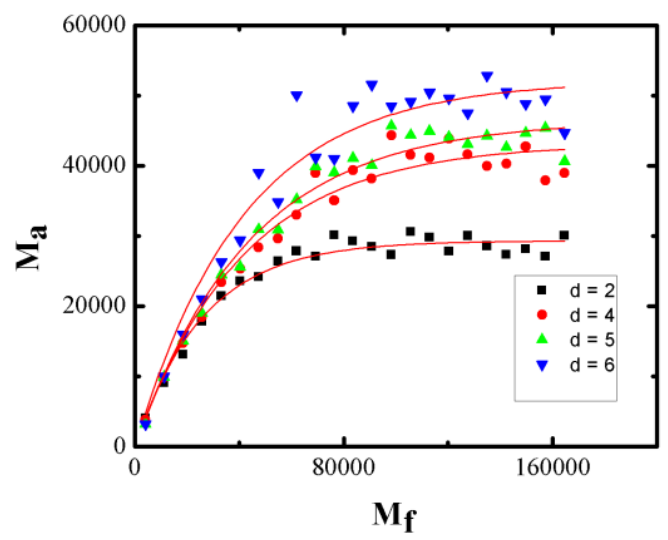

Figure 2. Evolution of apparent mass with the filling mass

This branching of data lines needed further investigation as bead size influence the stress transmission in a silo. The reason of such phenomenon lies in Janssen model, used in silo design [4]. It rests on following three assumptions. (1) Horizontal and vertical stresses are directly proportional to each other. (2) The forces between the beads and wall have attained the maximum values. (3) The medium is considered as continuous. These assumptions results in a model with the prediction that the mean pressure saturates after 
certain filling height. The equation relating the apparent mass and filling mass is given by

$$
M_{a}=M_{s}\left(1-e^{-M_{f} / M_{s}}\right) \ldots \ldots \text { (4) }
$$

where saturation mass is,

$$
M_{s}=\rho \pi(D / 2)^{3} /(2 \mu \mathrm{K}) \ldots \ldots \text { (5) }
$$

Mf is the filling mass, while material density is represented by $\rho$, and $\mu$ being the friction coefficient between grains and wall, the horizontal and vertical stresses ratio is denoted by $K$. Equation. 4 do not provide any relation regarding the bead size and saturation mass. However, it can be used to extract the values of saturation mass by simulating the data point lines. The solid lines depicted in Figure 2 are also obtained by using the equation 4 . It is illustrated that with the augment in grain diameter, the splitting of stress saturation curves occurs. The redirection of vertical stresses towards the confining boundary is the main reason for such variation.

The splitting of data lines with augment in " $\mathrm{d}$ " may be due to the reason that conversion of vertical to horizontal stresses is reduced with the augment in bead size. It implies that when granular column comprises of smaller bead sizes there is strong tendency of conversion of vertical stress into the horizontal ones. Thus redirection parameter $\mathrm{K}$ is efficient in such configurations. The reason lies in the fact that number of contact points between the beads and silo is reduced in the presence of larger size of grains. Hence more stress is propagated to the base of silo rather than to the side wall. Consequently the screening effect is minimized in the case of larger size of grains and higher saturation mass values are obtained. It appears that friction between grains and the silo wall leads to the variation in apparent mass.

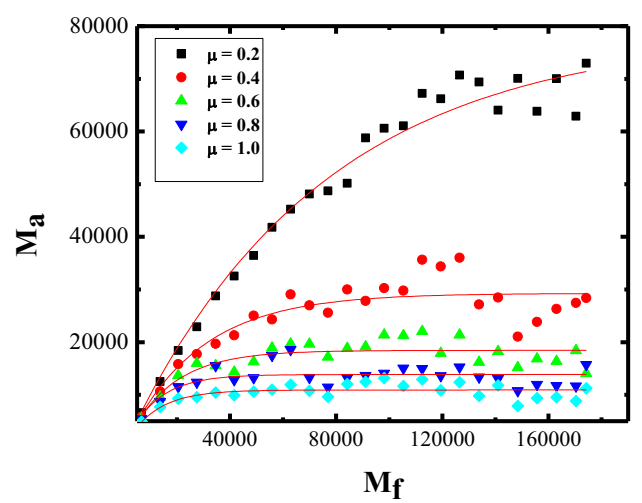

Figure 3. Variation of apparent mass with filled mass for various co-efficient of friction

To further elaborate the role of friction in variation of apparent mass, the simulations are run for the same bead diameter while the friction coefficient between the grains and the silo wall has been varied. When the grain diameter is $\mathrm{d}=2.0$ units the friction coefficient is changed from $\mu=0.200 .40,0.60,0.80$ to 1.00 . It can be observed from the Figure 3 that augmentation of friction values results in minimizing the apparent mass. Figure 3 also shows that for, $\mu=0.20$ the granular material exhibits hydrostatic behavior in a silo. The behavior does not alter even for different grain sizes. However in this paper results of bead diameter $d=2$ units are presented. Generally, it is assumed that screening effect in granular media is due to the arching [19]. However, the presented research using different diameter of grains in a silo it is revealed that in addition to the arching, the friction also plays an important role in shielding of weight in silos.

\section{Conclusion}

Large-scale simulations have been carried out to measure the mean vertical pressure at the base of cylindrical granular pile. It is shown that the classical Janssen analysis predicts the saturation of mass in a granular column also as observed in the simulation. It is found that the shielding effect is a macroscopic one and it should not be attributed only to the arching, but also to the friction between grains and the confining wall of the silo. Furthermore, it has been observed that the stress transmission at the bottom of packing also depends upon the size of grains. In future this work will be corroborated with the role of number of contact point at different conditions on the apparent mass so as to further elaborate the screening effect. Thus, more analyses such as the averaged normal force and tangential force can be carried out.

\section{Acknowledgement}

The funding for this project has been provided by Sukkur Institute of Business Administration, Sindh, Pakistan.

\section{References}

1. Ram Chand, Abdul Qadir, Shi Qing-Fan, Zheng Ning, and Sun Gang. 2011, Chin. Phys. Lett., 28(9): 098301.

2. Abdul Qadir, Madad Ali Shah, and Saeed Ahmed Khan. 2013, Chin. Phys. B, 2013, 22(5): 058301.

3. Li Zhi-Feng, Peng Zheng, and Jiang Yi-Min. 2014, Acta Physica Sinica, 63(10): 104503.

4. Janssen H and Vereins A Z 1895 Dtsch. Eng. 39 1045.

5. Vanel L, Claudin Ph, Bouchaud J P, Cates M E, $\mathrm{Cl}^{\prime}$ ement E and Wittmer J P 2000 Phys. Rev. Lett. 847

6. Cundall P A and Strack O D L, 1979, Geotechnique, 29: 47-65.

7. Bertrand F, Leclaire L.A, and Levecque. G, 2005, Chem. Eng. Sci., 60: 2517-2531.

8. Matuttis H G, Luding S, and Herrmann H J, 2000, Powder Technology, 109, 278-292

9. Hong D.C, Quinn P V, and Luding S, 2001, Phys. Rev. Letts., 86, 3423-3426.

10. Zheng He-peng, and Jiang Yi-min, 2008, Chin. Phys., 57(12):7919-7927. 
11. Liu J, Shi Q, Xuanwen L, Lei Y and Sun G 2009 Physica A 388379.

12. Bruni Gilles, Emmanuel Leveque, Claude laroche and Christophe coste, 2004 Phy. Rev. Lett. 92, 20.

13. Landry, J.W., Grest, G.S., Silbert, L. E. and Plimpton, S.J, 2003, Physical Review E, 67, 4.

14. Landry, J.W., Grest, G.S., and Plimpton, S.J., 2004, Powder Technology, 139, 233, 2004.

15. Kloss C, Goniva C, Hager A, Amberger S, and Pirker S, 2012, Prog. Comput. Fluid Dyn. 2, 3.

16. Kloss C, and Goniva C, LIGGGHTS - A new open source discrete element simulation software. Proc. of the fifth International Conference on Discrete Element Methods, London, UK, ISBN 978-0-9551179-8-5, August 2010, pp. 25-26.

17. Plimpton S, 1995, J. Comp. Phys. 117 1-19. Web-site of the project: http://lammps.sandia.gov/.

18. Kadanoff L P, 1999 Rev. Mod. Phys. 71, 435. 